\title{
Contribution du traitement des images-satellite à la cartographie des pâturages sahéliens
}

\author{
par G. DE WISPELAERE (*) et G. WAKSMAN (**)
}

\begin{abstract}
RÉSUMÉ
Certains pâturages sahéliens se sont considérablement dégradés au cours des années de sécheresse 1972-1973. Face à cette situation, il est devenu indispensable de procéder à d'importants travaux d'inventaires actualisés des ressources fourragères. Ces inventaires concernent des superficies de plus en plus vastes et s'orientent vers l'étude de l'évolution des parcours.

Les images-satellite offrent une solution à quelques-uns des problèmes nouveaux posés par l'étude de régions immenses à des pérıodes successives. Mais l'interprétation des images-satellite exige une approche globale au travers d'unités synthétiques : les Unités Paysages Pastorales.

Traitées par informatique à $1 / 100000$, les images-satellite permettent d'importants travaux de reconnaissance. Employées conjointement avec des observations de terrain et des photographies aériennes, elles rendent possible la cartographie actualisée des ressources pastorales.
\end{abstract}

\section{INTRODUCTION}

\section{La cartographie des pâturages}

Les études de pâturages naturels tropicaux peuvent être classées en deux grandes catégories $(16,17)$ :

- les inventaires de ressources pastorales au niveau national ou régional, plus particulièrement destinées aux planificateurs ;

- les études ponctuelles dans le cadre des projets de développement d'élevage intensif (ranches d'embouche ou de réélevage).

La synthèse des observations et des mesures est présentée le plus souvent à l'aide de cartes qui appartiennent au groupe des cartes de végétation dont elles diffèrent cependant en représentant plus particulièrement les espèces fourragères dominantes. Elles indiquent donc :

(*) Institut d'Elevage et de Médecine Vétérinaire des Pays Tropicaux, 10, rue Pierre-Curie, 94700 MaisonsAlfort (France).

(**) Compagnie Générale de Géophysique, B. P. 56, 6, rue Galvani, 91301 Massy (France).

(C) I. E. M. V. T. - C.G.G. 1977.
- la nature et la répartition des principales espèces constituant le pâturage ;

- la biomasse fourragère et la capacité de charge pour chaque type de pâturage retenu dans l'inventaire ;

\section{- les périodes d'exploitation optimale.}

L'information sur la végétation est complétée par les indications nécessaires à la gestion pastorale, points d'eau, cures salées, infrastructure vétérinaire.

Enfin, l'utilisation des cartes de pâturages est facilitée par sa superposition sur un fond topographique aussi récent que possible.

\section{L'évolution des besoins}

Au Sahel, le paysage végétal peut évoluer rapidement, lorsque de brusques variations de pluviosité viennent perturber l'équilibre précaire qui règne dans ces régions $(5,21)$. Ce phénomène a été particulièrement sensible après la sécheresse des années 1972 et 1973. Celle-ci provoqua une diminution considérable du stock 
fourrager et entrainna la disparition d'un pourcentage parfois très élevé du cheptel bovin $\left(^{*}\right)$. Les aléas climatiques n'agissent pas seuls sur le paysage végétal; 1'homme avec ses cultures, ses animaux et ses feux, intervient également d'une façon plus brutale qu'on ne l'imagine généralement (15).

- Ces transformations ont, en partie, modifié les objectifs des études agropastorales. De descriptives et statiques, elles se sont progressivement transformées, pour devenir dynamiques par l'étude et la cartographie des évolutions (18). Simultanément à cette modification des objectifs, les inventaires ont concerné des superficies de plus en plus vastes et ont dû être réalisés dans des délais de plus en plus courts.

- Face à cette évolution des besoins, il était indispensable d'adapter les méthodes, d'autant qu'avec les satellites de ressources terrestres, des projets de surveillance continue des pâturages (monitoring) ont pu être envisagés (9).

\section{L'expérimentation ; sa localisation}

La présente expérience s'est fixée pour but de préciser l'apport des images-satellite comparé à celui des photographies aériennes classiques dans un cadre de cartographie pastorale. Pour être probante, cette recherche exigeait :

- des observations récentes concernant l'écologie du périmètre retenu ;

- une couverture photographique aérienne récente dont la date de prise de vue coïncide le plus exactement possible avec celle de l'image-satellite.

Ces conditions se sont trouvées remplies en Haute-Volta dans la région de l'Oudalan où l'Institut d'Elevage et de Médecine vétérinaire des Pays tropicaux réalise pour la Direction des Services de l'Elevage et des Industries Animales, l'étude et la cartographie des pâturages de l'Office Régional de Développement du Sahel et de la zone de délestage au Nord-Est de Fada N'Gourma (33).

Pour réaliser ce travail, l'I. E. M. V. T. dispose de l'ancienne couverture photogra-

(*) M. GARCIA (20) estime que dans le cercle de l'Oudalan en Haute-Volta les pertes dues à la sécheresse ont pu atteindre jusqu'à 62 p. 100 alors que le pourcentage global des pertes pour les régions étudićes est estimé à 26 p. 100 . phique à 1/50 000 émulsion panchromatique de novembre 1955 , d'une prise de vue également à $1 / 50000$ en deux émulsions panchromatique (P) et infrarouge noir et blanc (I. R.) de novembre 1974, et enfin sur l'emplacement de la zone test, d'une reprise du 8 novembre 1975 toujours à $1 / 50000$ en $\mathbf{P}+$ I. R.

Les traitements informatiques ont été réalisés par la Compagnie générale de Géophysique à partir de la bande magnétique du satellite LANDSAT 2 de la N. A. S. A. du 6 novembre 1975 (scène $\mathrm{n}^{\circ} 2$ 288-09 403).

Les observations de terrain pour l'ensemble de la zone d'étude se sont déroulées d'octobre 1974 à novembre 1976, le site lui-même ayant été étudié à plusieurs époques, dont une en février 1976 lors de l'étalonnage des photographies aériennes de 1975.

La zone retenue pour l'expérimentation (fig. 1) se situe par $14^{\circ} 20^{\prime}$ de latitude Nord et $\mathrm{O}^{\circ} 35^{\prime}$ de longitude Ouest entre Aribinda et la mare d'Oursi.

\section{LES SATELLITES LANDSAT A ET B}

Les 2 satellites LANDSAT fournissent de par leurs caractéristiques une vue entièrement nouvelle de la terre. A $920 \mathrm{~km}$ d'altitude, ils suivent une orbite quasi circulaire et sub-polaire telle que chaque satellite passe sur le même point tous les 18 jours. L'ensemble des images enregistrées par les deux satellites permet donc d'avoir une vue de la même surface à la même heure tous les neuf jours.

Ces images sont recueillies dans des conditions très homogènes puisqu'une superficie d'environ $185 \mathrm{~km} \times 185 \mathrm{~km}$ est analysée en une vingtaine de secondes par un radiomètre multispectral à balayage (Scanner).

Ce scanner (fig. 2) est un appareil de mesure optique, dont le miroir oscillant balaye le sol point par point, le long d'une ligne perpendiculaire à la direction de l'avancement du satellite puis ligne par ligne du fait du déplacement de ce dernier. L'image-satellite se constitue donc point par point et présente plus d'analogies avec une image de télévision qu'avec une photographie. 


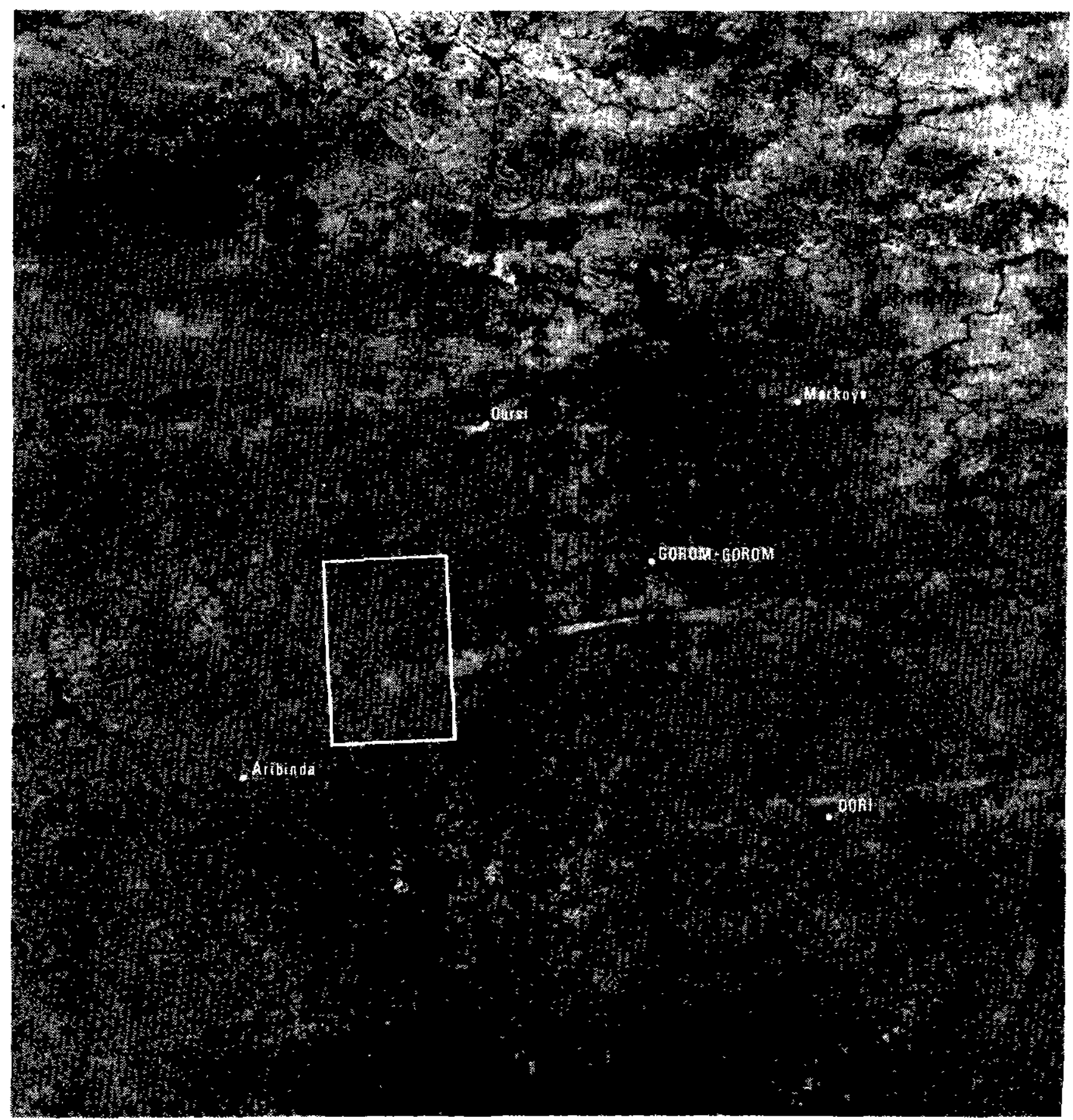

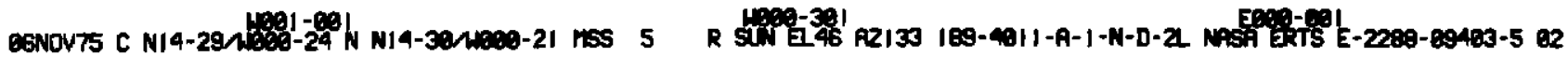

Fig. 1 : Tirage à 1/1.000.000 du canal 5 de la scène

LANDSAT 2 : 2289-09403 du 06 Nov. 1975

Localisation de la zone test. 
hadiometre multispectral a balayage

(SCANMER)

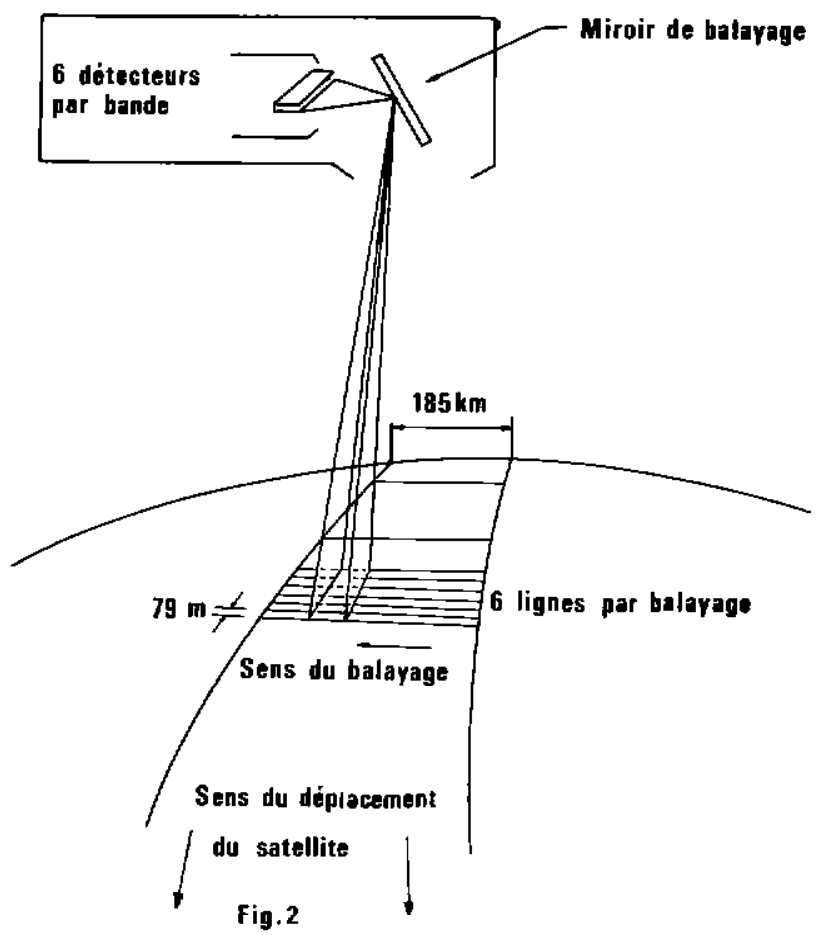

Pour chacun des points examinés, c'est-à-dire pour chaque élément de résolution (ou pixel) du scanner, celui-ci mesure quatre intensités lumineuses. Cette mesure d'intensité lumineuse réfléchie par le sol est effectuée dans 4 longueurs d'onde qui correspondent à peu près au vert jaune (canal 4), au jaune orangé (canal 5), au rouge (canal 6) et à l'infrarouge proche (canal 7).

Les satellites LANDSAT transmettent directement par radio ces intensités, dès qu'ils entrent en vue d'une antenne réceptrice, c'està-dire dans un rayon de $3000 \mathrm{~km}$ autour de cette dernière ; dans ce cas, la fréquence d'acquisition est de 18 jours. Ailleurs, la capacité de mémoire des satellites étant limitée, cette fréquence devient plus incertaine et généralement plus lente.

Dans les stations réceptrices, les données sont stockées sur bandes magnétiques puis visualisées sous forme d'images noir et blanc à l'échelle du 1/3 339000 , à partir desquelles sont produits des agrandissements photographiques.

\section{LE TRAITEMENT INFORMATIQUE DE L'IMAGE-SATELLITE}

Les échelles des meilleurs agrandissements photographiques, réalisables seulement avec d'excellents originaux, se situent à $1 / 250000$ environ. Mais ces échelles restent insuffisamment précises pour les utilisateurs. De plus au cours des reproductions photographiques successives, la qualité décroît, alors que l'information est entièrement conservée lors de la copie d'une bande magnétique.

Les deux objectifs essentiels du traitement informatique des données-satellite sont :

- l'amélioration des contrastes ;

- la production d'une image à $1 / 100000$ corrigée géométriquement.

Pour chaque pixel, on fait correspondre à l'intensité mesurée par le scanner du satellite dans chaque longueur d'onde, un niveau de gris. Ce dernier est choisi dans une gamme de niveaux de gris préalablement définie. L'amélioration des contrastes est réalisée, comme sur photographie, par étapes successives, en répartissant les niveaux de gris disponibles de façon 
à obtenir une image où aucun niveau ne domine et où les unités de paysages, auxquelles s'intéresse l'utilisateur, ressortent au mieux.

Les corrections géométriques sont effectuées par un traitement d'interpolation qui ajuste les images aux cartes existantes. Il s'agit ici de corriger les effets des variations d'altitudes et d'attitude du satellite (lacé, roulis, tangage), et de se ramener à une projection cartographique définie. La précision géométrique de l'image corrigée est alors celle de la carte. L'image à $1 / 100000$ corrigée sur la base de la carte à $1 / 200000$ a la précision planimétrique du $1 / 200000$, mais contient du point de vue du photo-interprète, beaucoup plus de détails que la carte.

\section{METHODE D'APPROCHE POUR L'INTERPRETATION}

Sur les photographies aériennes et sur les images satellites, les objets diffèrent par leur forme et leur densité. La densité exprime la quantité d'énergie enregistrée par le capteur ; énergie que l'on peut assimiler à celle réfléchie, ou émise, par les objets examinés. Ainsi, sur une photographie panchromatique, un objet lumineux est traduit par un gris clair (faible densité) et un objet sombre est représenté par un gris noir (forte densité). Mais la relation entre un objet et la quantitê d'énergie enregistrée dépend de l'éclairement, de l'état de cet objet et de la qualité de l'enregistrement (11, 27).

Les photographies aériennes permettent, grâce à leur haute résolution et à la vision stéréoscopique du relief, l'observation détaillée des formes. Les images-satellite, avec leurs faibles résolutions $(79 \mathrm{~m} \times 57 \mathrm{~m})$ et leur absence de stéréoscopie, sont d'une approche plus difficile.

Que la prise de vue soit réalisée par avion ou enregistrée par satellite, l'information recueillie est globale et tous les constituants superficiels du paysage interviennent dans la quantité d'énergie enregistrée ; que ce soient les affleurements rocheux, les sols plus ou moins humides ou la végétation avec ses différents niveaux de couvert et ses différents stades végétatifs. I semble donc difficíle, sinon ímpossible, de traiter une composante séparément des autres, la végétation sans les sols ou inversement (23).
Dans la région étudiée, le couvert végétal est une steppe arbustive, composée d'une strate herbacée dont le recouvrement varie de 25 à 80 p. 100 et d'une strate ligneuse très claire en moyenne, avec moins de 10 arbustes à l'ha. Lorsque le couvert végétal est inférieur à 30 p. 100 environ, on constate que la réflectance du paysage tient en premier lieu au sol. Dans ce secteur climatique, la période la plus favorable aux enregistrements se situe lorsque la végétation herbacée a atteint son plein développement vers les mois d'août ou septembre. Mais à ces périodes, la couverture nuageuse interdit la réalisation de prises de vues. Il faut donc se contenter des enregistrements de début de saison sèche (octobre dans ces régions), époque où les graminées annuelles sont toutes sèches et sans contraste avec le sol.

Cette situation rend l'interprétation de la végétation obligatoirement indirecte, par le biais d'entités désignées sous le terme d'Unités Paysages Pastorales (U.P.P.) (*). La qualité de l'interprétation dépendra donc directement des relations qui seront établies par l'étude de terrain entre les pâturages et les principaux facteurs édaphiques.

\section{EXPLOITATION DU TRAITEMENT DES DONNEES-SATELLITE}

Le résultat du traitement informatique des données-satellite est visualisé par quatre images, une par canal, en noir et blanc à l'échelle du 1/100 000 (fig. 3).

L'échelle la plus petite qui puisse être obtenue sans problèmes en photographies aériennes, se situe aux alentours du 1/80000. A l'autre extrémité, les agrandissements photographiques des images-satellite permettent d'obtenir des photographies de bonne qualité à $1 / 1000000,1 / 500000$ et quelquefois à $1 / 250000$. La présente méthode fournit une image corrigée géométriquement à $1 / 100000$ éventuellement réductible à $1 / 200000$, comblant ainsi un créneau entre ces deux ćatégories d'échelles.

(*) U. P. P. : Unité territoriale identifiable à tous les niveaux d'observation, souvent désignée par un terme géomorphologique ou édaphique qualifié par un type de physionomie végétale et précisant les dominantes fourragères (9). Cette entité s'apparente à celle du géofaciès de BERTRAND (4). 


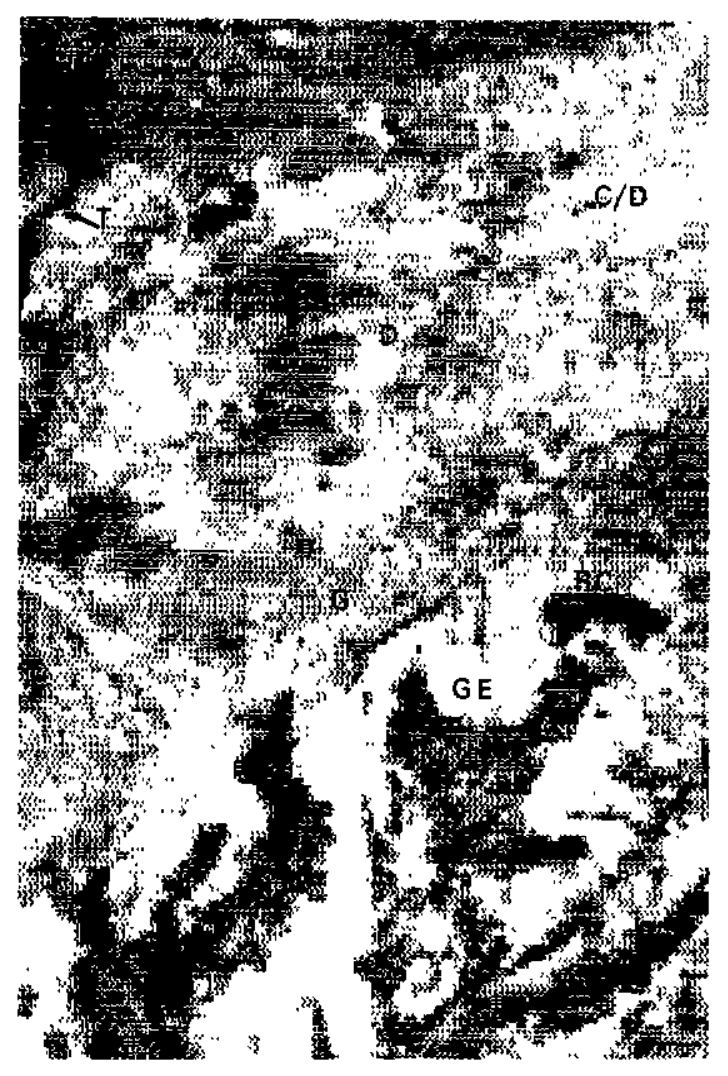

Canal 4

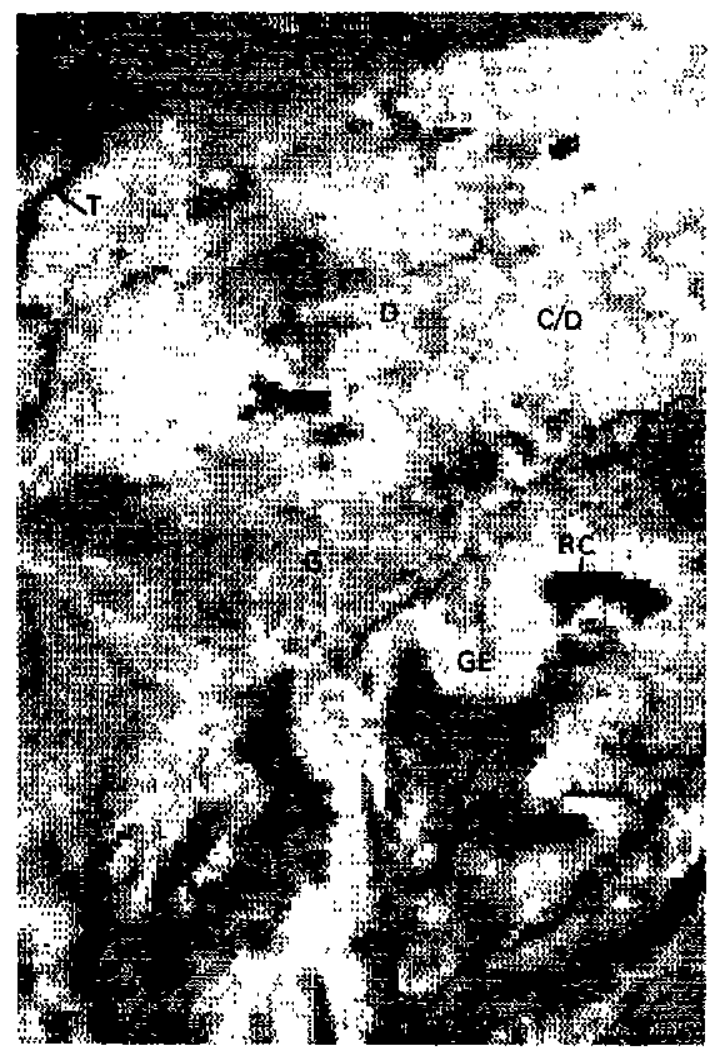

Canal 6

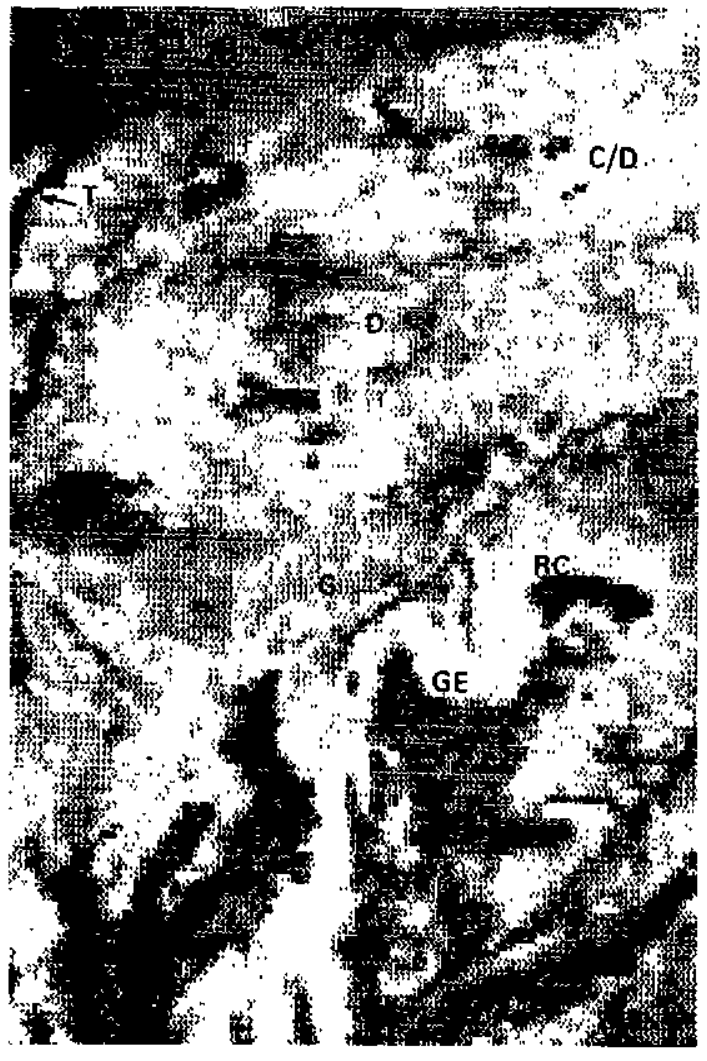

Canal 5

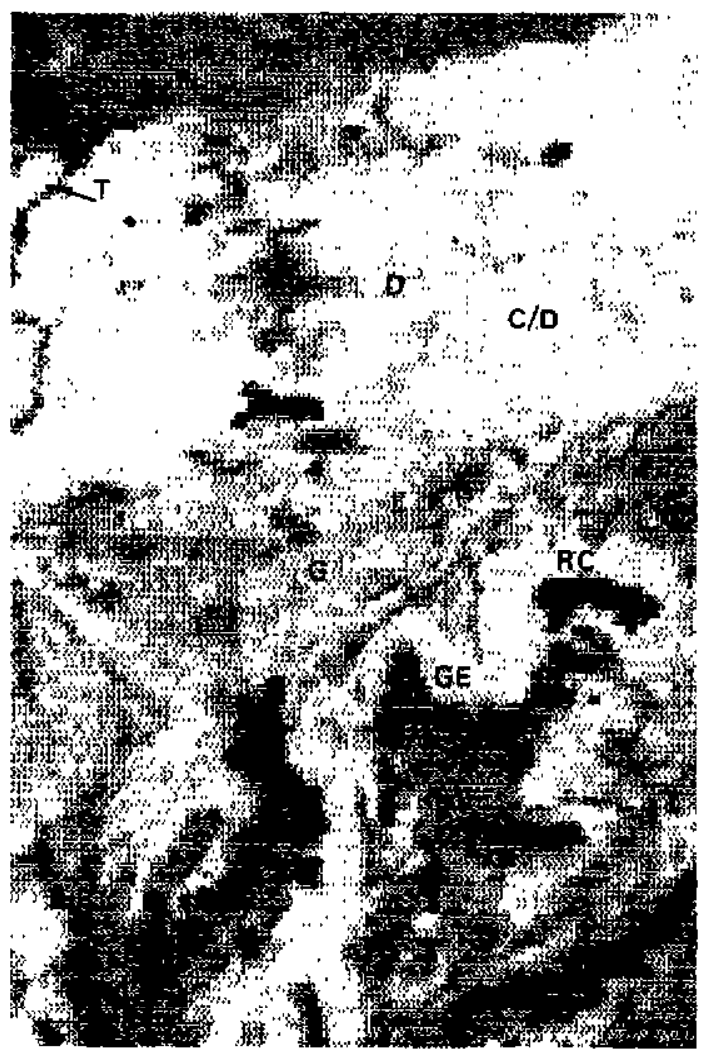

Canal 7

Fig. 3 : Visualisation du traitement informatique par canal séparé. 


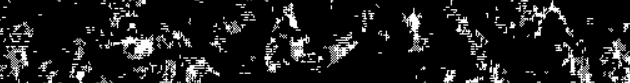
H

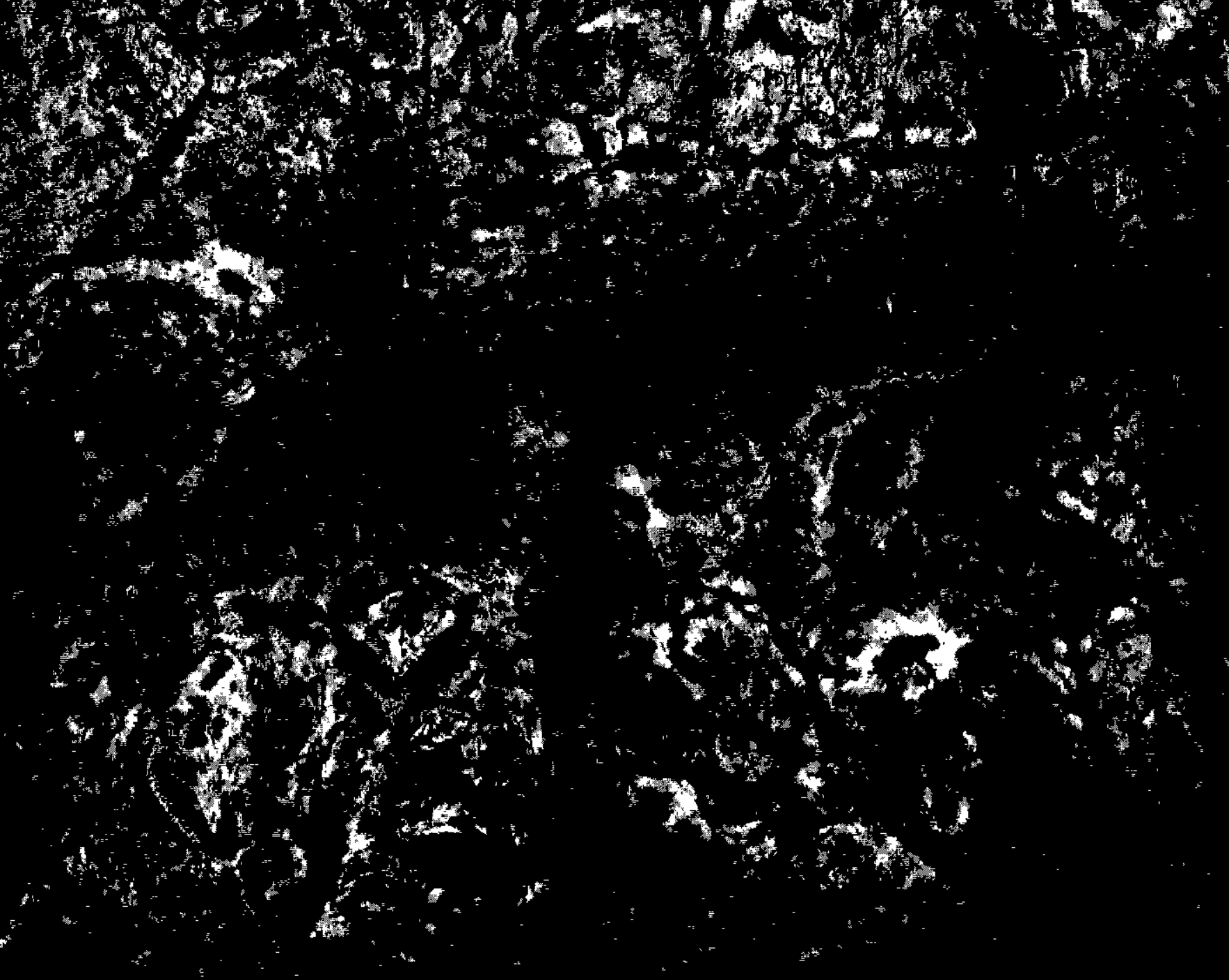

3
3
4
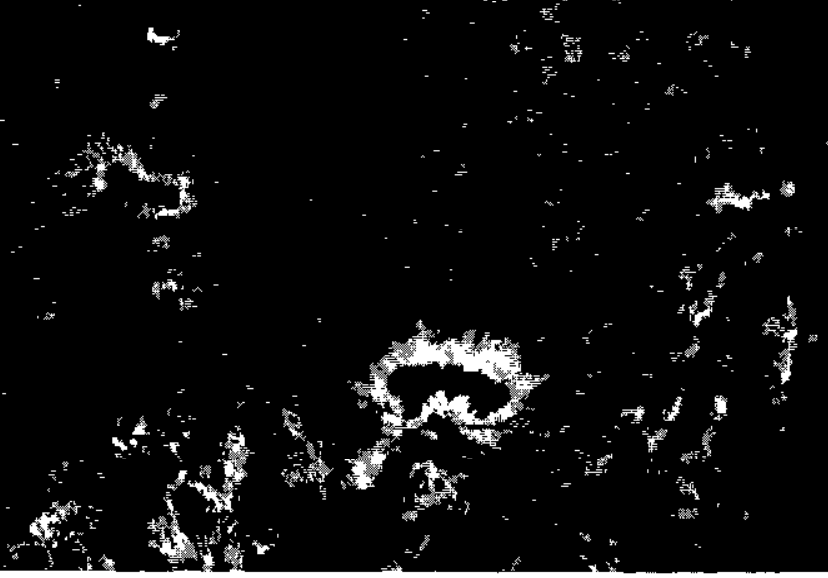

Fig. 4 : Réduction à 1/100.000 d'un assemblage de photographies aériennes, émulsion infra-rouge à 1/50.000, 
V. 1. Interprétation par canaux séparés (fig. 3)

Tout d'abord il a été procédé à une interprétation «canal par canal » c'est-à-dire sur chacune des images noir et blanc des canaux 4 , 5 , 6 et 7 . Ce travail s'est révélé long et peu concluant. En effet, la comparaison avec la mosaïque des photographies aériennes, réduite à $1 / 100000$ (fig. 4), montre que l'on retrouve sur chaque canal les limites plus ou moins nettes de chaque a Unité Paysage ".

La différence de contraste entre les sols sablonneux cultivés (C/D) et les glacis limonoargileux (G) est plus nette dans le canal 7 que dans les autres. Pour le canal 4 , on observe le même phénomène sur les glacis d'érosion (G. E.) des buttes cuirassées ferrugineuses. Quant aux rochers cuirassés eux-mêmes (R. C.), ils sont parfaitement discernables sur l'ensemble des canaux, ainsi d'ailleurs que le thalweg (T) coupant le cordon dunaire (D).

\section{2. Interprétation de la composition colorée (fig. $5 \mathrm{~A}$ et $5 \mathrm{~B}$ )}

L'exploitation des images par canaux séparés noir et blanc n'utilise pas le caractère multispectral des données. Des compositions colorées ont alors été réalisées en superposant 3 ou 4 canaux avec pour chaque canal une couleur arbitraire, conventionnelle ou non.

La difficulté majeure tient au fait que deux paysages de nature ou d'état différents peuvent avoir la même expression aussi bien sur photographie aérienne que sur image-satellite. Inversement, deux paysages jugés identiques sur le plan pastoral peuvent être exprimés de manière

TABLEAU $\mathbb{N}^{\circ} \mathrm{I}$

\begin{tabular}{|c|c|c|c|c|c|}
\hline $\begin{array}{c}\text { Unite } \\
\text { Payrege (1) }\end{array}$ & $\begin{array}{c}\text { Faciès } \\
\text { (1) }\end{array}$ & $\begin{array}{c}E t a t \\
(1)\end{array}$ & $\begin{array}{c}\text { Canal } 4 \\
\text { (imprimé en jaune) }\end{array}$ & $\begin{array}{c}\text { Canal } 5 \\
\text { (en rouge) }\end{array}$ & $\begin{array}{c}\text { Canal } 7 \\
\text { (en bleu) }\end{array}$ \\
\hline \multirow{3}{*}{$\mathbf{S}$} & \multirow[b]{2}{*}{ SD } & SD & $x$ & $\mathbf{x x}$ & $x x x$ \\
\hline & & $\mathrm{C} / \mathrm{SD}$ & $\begin{array}{l}\text { variable } \\
\text { de } x \rightarrow 0\end{array}$ & $\begin{array}{r}\text { variable } \\
\text { de } \mathbf{x x} \rightarrow 0\end{array}$ & $\begin{array}{r}\text { variable } \\
\text { de } x \times x \rightarrow 0\end{array}$ \\
\hline & SE & $C / S E$ & $\begin{array}{l}\text { variable } \\
\text { de } x \rightarrow \mathbf{x x x}\end{array}$ & $\begin{array}{l}\text { variable } \\
x \text { et } x x\end{array}$ & $\begin{array}{c}\text { variable } \\
\text { de } x \times x+o\end{array}$ \\
\hline \multirow{2}{*}{$\mathbf{R}$} & $\mathrm{R} 1$ & - & $x x$ & $x$ & $x$ \\
\hline & $\mathrm{R} 2$ & - & $x x$ & $\mathbf{x}$ & $\mathbf{x x}$ \\
\hline \multirow{9}{*}{ G } & \multirow{3}{*}{ GP } & $\mathrm{GP}$ & $x$ & $\mathbf{x x x}$ & $\mathbf{x x}$ \\
\hline & & $\mathrm{GP} \ddot{ }$ & $x x$ & $x x$ & $x x$ \\
\hline & & $\mathrm{C} / \mathrm{GP}$ & $\begin{array}{c}\text { variable } \\
\text { de } x \rightarrow \operatorname{sxx}\end{array}$ & $\begin{array}{l}\text { variable } \\
x \text { et } x x\end{array}$ & $\begin{array}{l}\text { variable } \\
\text { de } x \times x \rightarrow 0\end{array}$ \\
\hline & \multirow{2}{*}{ G1 } & G1 & $x x$ & $x x x$ & $\mathbf{x}$ \\
\hline & & G1:: & $x x$ & 0 & $x x$ \\
\hline & \multirow{2}{*}{ G2 } & G2 & $x x$ & $x x$ & $x$ \\
\hline & & $\mathrm{G} 2{ }^{2}$ & $x \times x$ & $x x$ & $x$ \\
\hline & \multirow{2}{*}{ G3 } & G3 & $x x$ & $\mathbf{x}$ & $x x x$ \\
\hline & & G3: & $\begin{array}{l}\text { variable } \\
\text { de } x \mathbf{x}+\mathbf{0}\end{array}$ & $\begin{array}{r}\text { variable } \\
\text { de } x x \rightarrow 0\end{array}$ & $\mathbf{x x K}$ \\
\hline \multirow{4}{*}{ v } & \multirow{3}{*}{ Vl } & V1 & $x x$ & $x$ & $x x$ \\
\hline & & $\nabla 1 x$ & $x x$ & $\mathbf{x x}$ & $\mathbf{x x x}$ \\
\hline & & $\mathrm{C} / \mathrm{V} 1$ & $x$ & $x$ & $x x K$ \\
\hline & v2 & & $\mathbf{x}$ & $\mathbf{x}$ & $x \times x$ \\
\hline
\end{tabular}

(1) Pour la signification des symboles, se reporter au tableau $n^{\circ}$ IV

$x$ - forte coloration indiquant une réflectance faible (et/ou absorption dans l'infra-rouge du canal 7). $x x$ - coloration moyenne traduisant une réflectance moyenne.

$x x x$ - faible coloration exprimant une réflectance forte.

- - absence de couleur indiquant une saturation (ou une très forte réflectance). 
différente. La première ambiguité est très fréquente et la plupart du temps seule la prospection de terrain permet de la lever. La seconde est plus rare et tient le plus souvent à l'agencement différent des végétaux de même espèces.

Les combinaisons colorées obtenues mettent en évidence certaines relations entre les « Unités Paysages » (U. P.) et leurs réflectances (tabl. I). Ainsi l'U. P., Cordons dunaires et dépôts sableux (S) est traduite par une réflectance faible dans les canaux 4 iet 5 (dominance de jaune et de rouge) et forte dans le canal 7 (faible présence de bleu).

L'U. P. Inselberg et sols squelettiques (R) se voit exprimée par une coloration plus ou moins noirâtre résultant de l'addition des trois couleurs, ce qui indique une faible réflectance pour tous les canaux.

L'U. P. Glacis (G) est très hétérogène, sans caractère chromatique dominant, sauf pour le faciès G. P. qui se distingue par une coloration de tendance jaune, montrant des similitudes avec l'U. P. Cordons dunaires et dépôts sableux, parce que le sol de ce faciès est de texture sablonneuse.

Quant à l'U. P. Vallées (V), elle se différencie par une coloration moyenne brun rouge qui caractérise une faible réflectance dans le canal 5 (dominance de rouge), une réfleotance moyenne à forte par les canaux 4 et 7 (présence de jaune et bleu). Le tableau I résume pour chaque canal, les réflectances estimées d'après l'intensité de couleur pour tous les faciès de la zone expérimentale.

Il faut remarquer que les traces de feux récents (en hachures sur l'interprétation) risquent d'être confondues avec des affleurements rocheux R1 ou R2. Cette situation paraît devoir limiter l'emploi de la méthode en zone de savane brûlée tous les ans, sauf si l'on arrive à trouver un enregistrement avant le début des feux courants.

L'examen de la composition colorée et la comparaison des représentations chromatiques des faciès cartographiés montrent les difficultés et les risques de confusion que l'on peut rencontrer. Les tableaux II et III mettent en évidence les différences relatives entre les expressions des faciès cartographiés à différents états d'après l'image-satellite d'une part et les photographies aériennes d'autre part.

L'état du couvert végétal a autant, sinon plus, d'importance que la nature des faciès.

Les états dégradés sont fréquemment confondus entre eux et dans certains cas extrêmes (très forte réflectance) on risque de les confondre avec les cultures (ex. G2* et R2, G1* et G3*).

Quatre combinaisons ont été essayées dans le but de mettre mieux en évidence la végétation.

Combinaison 1

Canal 4 bleu

5 vert

7 rouge

T.T-

Combinaison 3

$\begin{array}{rr} & \\ \text { Canal } 4 \text { bleu } & \text { Canal } 4 \text { jaune } \\ 5 \text { rouge } & 5 \text { rouge } \\ 7 \text { jaune } & 7 \text { bleu }\end{array}$

Les combinaisons 2 et 4 ont donné des résultats très voisins.

Déjà utilisée par l'U. S. Geological Survey, en raison de la ressemblance avec les photographies aériennes en infrarouge couleurs (I. R. C.), la combinaison 4 fut retenue.

Le procédé de visualisation après traitement informatique fournit une image dont les caractéristiques sont comparables à une trame photomécanique : grâce à cette particularité, la combinaison colorée choisie a pu être imprimée selon le procédé Offset (voir fig. $5 \mathrm{~A}$ ).

\section{3 Analyse de l'interprétation}

Le tableau IV donne la signification des symboles utilisés sur le calque de la figure $5 \mathrm{~B}$.

Les définitions des faciès cartographiés résultent des observations de terrain et de leur interprétation $\left(^{*}\right)$. Ces faciès se justifient par leurs caractères écologiques d'une part et par leur destination à des fins pastorales d'autre part. Leurs caractères sont des «moyennes » et peuvent varier localement.

L'identification des faciès et de leurs états sur l'image-satellite a été permise par l'interprétation des photographies aériennes correspondantes. En effet, s'il est possible de délimiter les taches de même coloration sur la composition colorée et dans certains cas d'identifier des

(*) Celles-ci ont été réalisées par B. TOUTAIN dans le cadre de l'étude des pâturages de l'O. R. D. du Sahel en Haute-Volta (33). 
TABLEAU II

Différences relatives entre les expressions des faciès cartographiés d'après l'image satellite

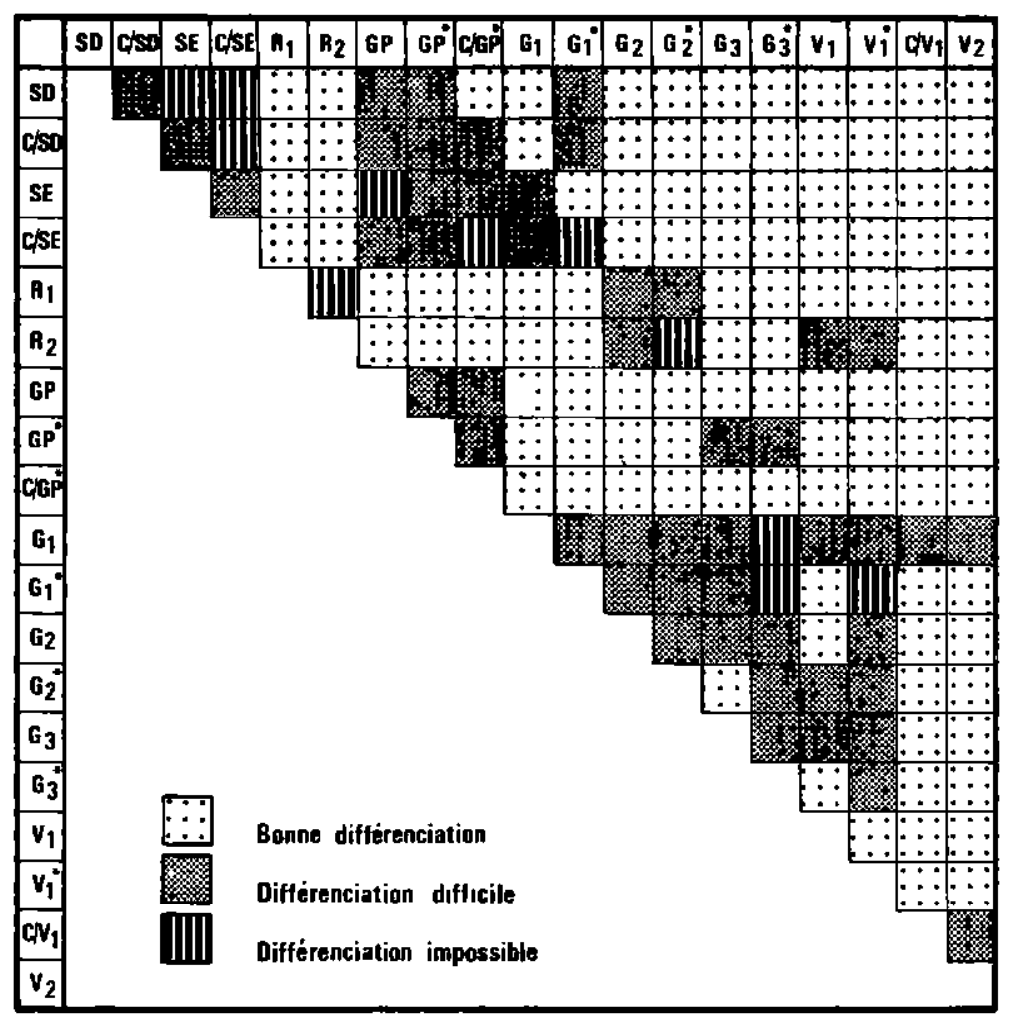

TABleaU III

d'après les photographies aériennes

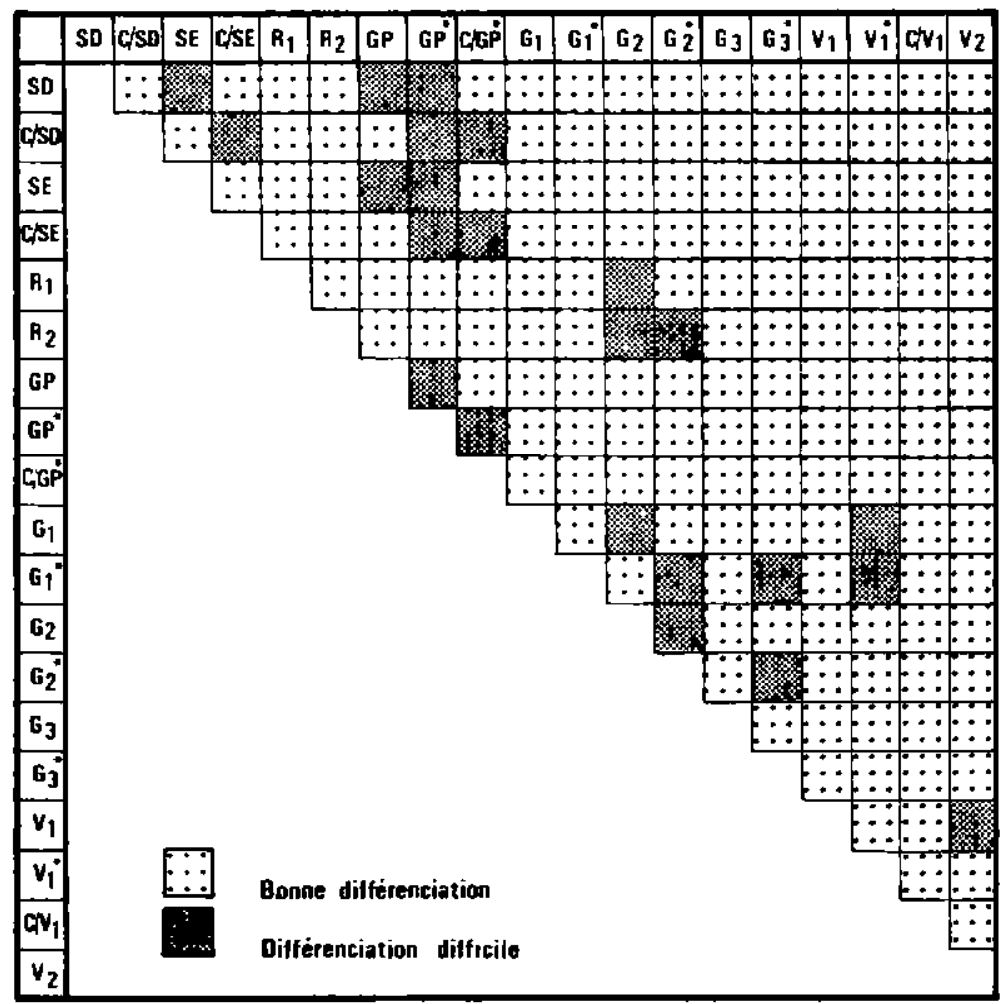


Tableau IV

\begin{tabular}{|c|c|c|c|c|c|c|c|c|c|c|c|c|c|}
\hline \multirow{4}{*}{ 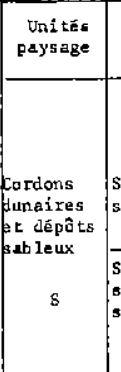 } & \multirow{3}{*}{$\begin{array}{c}\text { F a c i è s } \\
\\
\text { Steppe arbus tive } \\
\text { sur dune arasée }\end{array}$} & \multicolumn{2}{|c|}{$\begin{array}{c}\text { Eupèces végétales } \\
\text { domunantes }\end{array}$} & \multirow[t]{2}{*}{ वे } & \multicolumn{3}{|c|}{ Naveaux de dêtection couparés } & \multicolumn{3}{|c|}{$\begin{array}{c}\text { E tats du couvert végétal } \\
\text { Productivi té noyenne } \\
\text { des fourrages }\end{array}$} & \multicolumn{3}{|c|}{$\begin{array}{l}\text { Nive eux de détection } \\
\text { comporéa des états }\end{array}$} \\
\hline & & $\begin{array}{l}\text { strate } \\
\text { ligneuse }\end{array}$ & $\begin{array}{c}\text { Strote } \\
\text { herbacté }\end{array}$ & & $\begin{array}{l}\text { Observa- } \\
\text { tion au } \\
\text { sol }\end{array}$ & $\begin{array}{l}\text { Photogr. } \\
\text { aérienne } \\
1 / 50 \text { o00 }\end{array}$ & $\begin{array}{l}\text { Satellite } \\
1 / 100000\end{array}$ & (1) tet & $\begin{array}{l}\text { Produc tivi té } \\
\text { moyenne } \\
\mathrm{kg} \mathrm{MS} / \mathrm{ht} / \mathrm{an}\end{array}$ & pyobole & $\begin{array}{l}\text { Observat } \\
\text { tion au } \\
\text { sol }\end{array}$ & $\begin{array}{l}\text { Photogr. } \\
\text { aérieane } \\
1 / 50 \text { o00 }\end{array}$ & $\begin{array}{l}\text { Satellite } \\
1 / 200000\end{array}$ \\
\hline & & $\begin{array}{l}\text { Conbre tum } \\
\text { gtutinooum }\end{array}$ & $\left\{\begin{array}{l}\text { Cenchirus } \\
\text { bifitarus }\end{array}\right.$ & so & oui & our & $\begin{array}{l}\text { Différencia- } \\
\text { ti on }\end{array}$ & $\begin{array}{l}1 \\
2 \\
3\end{array}$ & $\begin{aligned} 1500 \text { a } & 2000 \\
500 \text { à } & 1000 \\
\vdots & \end{aligned}$ & $\begin{array}{l}\mathrm{SD}(2) \\
\mathrm{SD} \cdot(2) \\
\mathrm{C} / \mathrm{SD}\end{array}$ & oui & oui & $\begin{array}{l}\text { Différenciation } \\
\text { dëlicate avec } \\
\text { les jachères }\end{array}$ \\
\hline & $\begin{array}{l}\text { Steppe arbustive } \\
\text { sur épandage } \\
\text { sab lonneux }\end{array}$ & $\begin{array}{l}\text { Acacta } \\
\text { raddicana }\end{array}$ & $\begin{array}{l}\text { Cencinsua } \\
\text { biflorus }\end{array}$ & SE & oui & $\begin{array}{c}\text { oui } \\
\text { (délicate) }\end{array}$ & is & \begin{tabular}{l|l}
1 \\
2 \\
3
\end{tabular} & $\begin{array}{c}2400 \text { a } 3200 \\
\text { monns de } 1000 \\
:\end{array}$ & SE (2) & $\begin{array}{l}\text { oul } \\
\text { oui } \\
\text { oui }\end{array}$ & délicete & $\begin{array}{l}\text { oui } \\
\text { Diffërenciation } \\
\text { de C/D cmosos- } \\
\text { sible }\end{array}$ \\
\hline $\begin{array}{l}\text { Inselbergs } \\
\text { et sols } \\
\text { pqueletta- } \\
\text { ques }\end{array}$ & $\begin{array}{l}\text { Steppe arbustive } \\
\text { três claire sur } \\
\text { inselberg ro- } \\
\text { cheux } \pm \text { cui rasgé }\end{array}$ & varioble & très fanble & RI & oui & oui & $\begin{array}{l}\text { Différenciu- } \\
\text { ticn impossi- } \\
\text { ble suuf } \\
\text { certuins cas }\end{array}$ & \multicolumn{2}{|c|}{$\begin{array}{l}\text { Feu ou pas de } \\
\text { couvert, Intêrêt } \\
\text { pastoral nul }\end{array}$} & $\mathrm{Rl}$ & our & oui & our \\
\hline $\mathbf{k}$ & $\begin{array}{l}\text { Steppe arbustive } \\
\text { tres claire sur } \\
\text { df fleureurents } \\
\text { rocheux ou } \\
\text { gravillonnalres }\end{array}$ & variable & très faxble & $R 2$ & oul & oui & $\begin{array}{l}+ \text { confusion } \\
\text { avee G2 E } \\
\text { G2* }\end{array}$ & \multicolumn{2}{|c|}{$\begin{array}{l}\text { Couvert rrès faí } \\
\text { ble. In têrêt } \\
\text { pastoral rêduit }\end{array}$} & $R 2$ & ou1 & our & $\begin{array}{l}\text { Confusion } \\
\text { avec } 62 \%\end{array}$ \\
\hline \multirow[t]{4}{*}{ Glacis } & $\begin{array}{l}\text { steppe arbugtive } \\
\text { claire sur } \\
\text { piëmont sablon- } \\
\text { neur d'inselberg }\end{array}$ & $\left\{\begin{array}{l}\text { Acacia } \\
\text { raddiana } \\
\text { commiphora } \\
\text { afrioana }\end{array}\right.$ & $\begin{array}{l}\text { Sonoene fe lazic } \\
\text { gracilia } \\
\text { sristida } \\
\text { mutobilis }\end{array}$ & $\mathbb{P}$ & oui & $\begin{array}{l}\text { oui } \\
\text { Locale- } \\
\text { ment rig- } \\
\text { que de } \\
\text { confusion } \\
\text { avec D et } \\
\text { E }\end{array}$ & our & $\begin{array}{l}1 \\
2 \\
3\end{array}$ & $\begin{array}{c}2000 \div 3000 \\
1000 \\
:\end{array}$ & $\begin{array}{l}G P \\
G P: \\
C / G P\end{array}$ & $\begin{array}{l}\text { oui } \\
\text { oui } \\
\text { our }\end{array}$ & $\begin{array}{l}\text { oui } \\
\text { oui } \\
\text { oui }\end{array}$ & \begin{tabular}{l}
\multicolumn{1}{c}{ oui } \\
DiffErenciation \\
lmpossible + \\
confusion avec \\
C/D et $\mathrm{C} / \mathrm{E}$
\end{tabular} \\
\hline & $\begin{array}{l}\text { Sceppe arbustive } \\
\text { très clalre sur } \\
\text { glacis Iimono- } \\
\text { sableux }\end{array}$ & $\begin{array}{l}\text { Acacia } \\
\text { raddicona }\end{array}$ & $\begin{array}{l}\text { Sonoene fe titia } \\
\text { Gracitis }\end{array}$ & 61 & oui & \multirow{2}{*}{$\begin{array}{l}\text { D1ffërencia- } \\
\text { tion } \\
\text { délícate } \\
\text { par } \\
\text { endro2 ts }\end{array}$} & \multirow{2}{*}{$\begin{array}{l}\text { Différencia- } \\
\text { tion varıeble } \\
\text { selon l'état } \\
\text { du couvert }\end{array}$} & \begin{tabular}{l|l}
1 \\
2
\end{tabular} & hazns de 300 & G1: & $\begin{array}{l}\text { oui } \\
\text { our }\end{array}$ & $\begin{array}{l}\text { fou mais } \\
\text { ha dis- } \\
\text { tinction } \\
\text { des états }\end{array}$ & \multirow{2}{*}{$\begin{array}{l}\text { Diffétrenciation } \\
\text { des états dif- } \\
\text { Ficiles car en } \\
\text { plages diffuses. } \\
\text { G1: 1ocalement } \\
\text { confondus avec } \\
\text { G3: }\end{array}$} \\
\hline & $\begin{array}{l}\text { Steppe arbustive } \\
\text { claire sur glacis } \\
\text { pierreux lagno- } \\
\text { argileux }\end{array}$ & 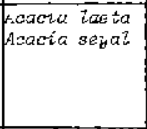 & 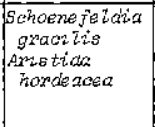 & $c_{2}$ & $\overrightarrow{\text { ou1 }}$ & & & \begin{tabular}{l|l} 
\\
2
\end{tabular} & $\begin{array}{r}700 \text { a } 2000 \\
\text { moins de } 300\end{array}$ & $\mathrm{G} 2$ & $\begin{array}{l}\text { oufi } \\
\text { ou1 }\end{array}$ & $\begin{array}{l}\text { pe peut } \\
\text { s'e Ifec- } \\
\text { tuer que } \\
\text { sur } 1 \text { 'er } \\
\text { pemble } \\
61+G 2\end{array}$ & \\
\hline & $\begin{array}{l}\text { Steppe arbustive } \\
\pm \text { dense sur sols } \\
\text { gravillonnal res } \\
\pm \text { sableux en } \\
\text { surface }\end{array}$ & $\begin{array}{l}\text { Ptercoarpus } \\
\text { incens } \\
\text { Combretum } \\
\text { gintinosum }\end{array}$ & $\mid \begin{array}{l}\text { Sohoene fe ldia } \\
\text { Gracilis } \\
\text { chroriz } \\
\text { prteurit }\end{array}$ & 63 & oui & oui & $\begin{array}{l}\text { oul mais } \\
\text { risque de } \\
\text { confusion } \\
\text { avec } V 2\end{array}$ & 1 & $\begin{array}{l}1000 \text { ₹ } 1500 \\
\text { woults de } 300\end{array}$ & G3 & oui & our & $\begin{array}{l}\text { Confum on avec } \\
\text { V1 } \\
\text { Confus1 on } \\
\text { aves G1\% }\end{array}$ \\
\hline \multirow[t]{2}{*}{$\begin{array}{c}\text { vallêes } \\
\mathrm{V}\end{array}$} & $\begin{array}{l}\text { Steppe arbugtive } \\
\pm \text { dense des sols } \\
\text { hydromorphes }\end{array}$ & Acoor $\alpha$ seyal & $\mid \begin{array}{l}\text { Panncum } \\
\text { Zas tum } \\
\text { Schoene fe lda a } \\
\text { gracizis }\end{array}$ & v1 & oui & ou1 & $\begin{array}{l}\text { aui wais } \\
\text { risque de } \\
\text { confusion } \\
\text { avec G3 }\end{array}$ & $\begin{array}{l}1 \\
2 \\
3\end{array}$ & $\begin{array}{c}1800 \text { a } 2500 \\
800 \\
x\end{array}$ & $\begin{array}{l}v 1 \\
v 1: \\
c v 1\end{array}$ & $\begin{array}{l}\text { sui } \\
\text { ou1 } \\
\text { oui }\end{array}$ & $\begin{array}{c}\text { oui } \\
\text { confusion } \\
\text { gvec ol } \\
\text { oui }\end{array}$ & \begin{tabular}{l}
\multicolumn{1}{c}{ oui } \\
Confusion avec \\
G1 \\
Confusion avec \\
V2
\end{tabular} \\
\hline & $\begin{array}{l}\text { Steppe arbustive } \\
\text { dense deg } \\
\text { vallees sab lon- } \\
\text { neuses }\end{array}$ & $\begin{array}{l}\text { pitiostigma } \\
\text { reticuiata } \\
\text { Givera } \\
\text { seregaiersis }\end{array}$ & $\begin{array}{l}\text { Penmise thm } \\
\text { pedice } 2 \text { ta thom } \\
\text { Andropogon } \\
\text { gaymus }\end{array}$ & $v_{2}$ & oux & oul & $\begin{array}{l}\text { yariable } \\
\text { sulvant la } \\
\text { densité du } \\
\text { couvert } \\
\text { lígneux }\end{array}$ & 1 & 1000 & v2 & oul & oui & $\begin{array}{l}\text { Confusion avec } \\
\mathrm{V} 1-\mathrm{G} 3-\mathrm{C} / \mathrm{E}\end{array}$ \\
\hline
\end{tabular}

(1) L'état de la végétation est exprimée selon la codification suivante :

1: Bon état et début de dégradation

2: Dégradation avancée : strate herbacée réduite, mort de nombreux ligneux

3 : Surfaces cultivées (cultures après récoltes et jachères).

(2) Situation qui n'existe pas sur la zone testée mais qui se rencontre ailleurs.

* Productivité variable : Sous-produits de récoltes.

Unités Paysages comme le cordon dunaire et les vallées, le plus souvent, la nature du «contenu» ne peut être précisée qu'avec les données de terrain et les clichés stéréoscopiques à $1 / 50000$.

\section{CONCLUSIONS}

Les conclusions que l'on peut tirer de cette expérimentation concernent principalement trois secteurs complémentaires.

\section{1) La visualisation des données-satellite}

En offrant aux pastoralistes une représentation multispectrale détaillée et précise d'importantes portions de terrains sans déformations géométriques, à des périodes différentes et à des échelles moyennes $(1 / 100000$ ou $1 / 200000$ ), le traitement des images-satellite ouvre des perspectives nouvelles en cartographie des pâturages. 


\section{2) Les techniques d'interprétation}

Bien que n'apportant pas autant d'informations que les photographies aériennes, les images-satellite présentent des avantages pratiques importants pour des travaux de reconnaissance et le report d'interprétation. Associée aux couvertures photographiques existantes et aux observations de terrain, l'image-satellite permet en outre une interprétation actualisée assez détaillée des Unités Paysages Pastorales.

Quand à la surveillance continue (Monitoring) des ressources fourragères pour laquelle la répétition des enregistrements des satellites constitue un atout majeur, peu de travaux ont exploré cette voie.

Cependant, les résultats présentés ici confirment que l'image-satellite constitue un bon «outil» pour la conception des cartes de références. Il reste à vérifier cependant que les évolutions éventuelles du paysage, à court et moyen terme, sont détectées par les satellites.

Enfin, l'extrapolation de la méthode à d'autres zones écoclimatiques reste à étudier.

\section{3) Diffusion des résultats cartographiés}

La visualisation colorée complétée par des informations topographiques sur la planimétrie, la toponymie et l'hydrographie constitue un véritable photo-plan en couleur, susceptible de recevoir en surimpression les limites et symboles des unités cartographiées. Cette procédure réduit de façon sensible les coûts d'édition cartographique.

Ainsi le traitement des données-satellite offre des possibilités réelles en matière de cartographie des ressources pastorales en région sahélienne, mais il est important de rappeler que la qualité d'une carte de pâturage tient essentiellement aux observations et mesures effectuées sur le terrain.

\section{SUMMARY}

\section{Contribution of satellite imagery processing to the sahelian pasture mapping}

Some range territories have considerably reduced during drought years 1972 and 1973. To cope with this situation, it has become necessary to undertake large scale range updated inventories. These laters deal with more and more huge surfaces and are directed towards range dynamic studies and monitoring.

Satellite-imagery may prove to be a solution to some of the new problems arisen from the study of huge areas at different periods. But satellite-image interpretation requires a total approach throught synthetic units which combine soil and vegetation characteristıcs : the Range Land Units.

When computer-processed at the $1 / 100000$ scale, the satellite-images enable large works of reconnaissance. Complementarily used with field observations and aerial photographs, this new tool allows to update the cartography of range resources.

\section{RESUMEN}

\section{Contribución del tratamiento de las imagenes por satélite a la cartografia de los pastos sahelianos}

Durante los años de sequia 1972-1973, se ha observado una degradación considerable de los pastos sahelianos. En dicha situación, se ha llegado a ser indispensable la realización de importantes trabajos de inventarios actualizados de los recursos forrajeros. Estos inventarios conciernen superficies cada vez más vastas y se orientan hacia el estudio de la evolución de los pasturajes.

Las imagenes por satélite ofrecen una solución a algunos de los nuevos problemas puestos por el estudio de regiones inmensas en periodos sucesivos. Pero la interpretación de las imagenes por satélite exige un acercamiento global por en medio de unidades sintéticas : las Unidades Paisajes Pastorales.

Tratadas por informatica a $1 / 100000$, las imagenes por satélite permiten importantes trabajos de reconocimiento. Es posible actualizar la cartografia de los recursos naturales empleandolas conjuntamente con observaciones de terreno $\mathrm{y}$ fotografias aereas. 


\section{BIBLIOGRAPHIE}

1. AMERICAN SOCIETY OF PHOTOGRAMMETRY. Manual of Remote Sensing. Falls Church, Va, U. S. A., 1975. 2 tomes. 2144 p.

2. BAUMGARDNER (M. E.). Evaluation of satellite data for use in natural resource surveys in Sudan. Rome, F. A. O., 1974, 47 p., 27 fig.

3. BAUMGARDNER (M. E.), KRISTOF (S. J.), SCHOLZ (D. K.). Identifying significant surface features in Upper Volta by computer. Implemented analysis of Landsat data L. A. R. S. W. Lafayette, Indiana, U. S. A., Purdue University, 1976, 7 p., 5 fig. 27 maps.

4. BERTRAND (G.). Paysage et géographie physique globale. Esquisse méthodologique. Rev. géogr. Pyr. Sud-Ouest, 1968 (39) : 249-72, 4 pl. h.t.

5. BOUDET (G.). Désertification de l'Afrique tropicale sèche. Adansonia, sér. 2, 1972, 12 (4) : 505-524.

6. BOUDET (G.). Manuel sur les pâturages tropicaux et les cultures fourragères. $2^{\mathrm{e}}$ éd., Paris, Minist. Coop., 1975, 255 p. (Coll. Manuels et Précis d'Elevage. 4$)$.

7. BOUDET (G.). Inventaire et cartographie des pâturages en Afrique de l'Ouest. in: Actes du Colloque ILCA. Inventaire et cartographie des pâturages tropicaux Africains, Bamako (Mali), 3-8 mars 1975. pp. 57-77.

8. BOUDET (G.), BAYENS (F.). Une méthode d'étude et de cartographie des pâturages tropicaux. Rev. Elev. Méd. vét. Pays trop., 1963, 16 (2) : 191-219.

9. BOUDET (G.), DE WISPELAERE (G.). Classification des pâturages tropicaux et niveaux de la télédétection. Rome, F. A. O., 1976.80 p., fig. en coul.

10. BRUNEAU (M.), LE TOAN (T.). Les clichés ERTS 1 et la cartographie des paysages en milieu tropical. Le cas de la Thaillande septentrionale. Bull. Soc. Fr. Photogrammétrie, 1976, 61: 39-55.

11. CAZABAT (C.). L'interprétation des photographies aériennes. Bull. Inf. IGN, 1969 (8) : 11-31.

12. CAZABAT (C). Recherche d'une méthodologie cartographique appliquée aux pâturages sahéliens. In : Actes du colloque ILCA : Inventaire et cartographie des pâturages tropicaux africains. Bamako, Mali, 3-8 mars 1975 , pp. 345-348.

13. C. E. G. E. T./C. N. R. S. Télédétection et environnement tropical. Réunion d'information. Talence, 18-19/4/1975. Talence, C. E. G. E. T., 1976, 171 p., tabl., fig., photogr. h. t, cartes.

14. DE WISPELAERE (G.). Applications de la télédétection à la cartographie des pâturages. Rapport de stage. Maisons-Alfort, I. E. M. V.T., 1974 $27 \mathrm{p}$.

15. DE WISPELAERE (G.). Carte d'occupation des sols du Sahel Voltaïque. Paris, Minist. Coop., 1976 (Méth, de Planif. du Dévelop. rural), pp. 15-16, 1 carte 3 feuilles $1 / 200000$.

16. DE WISPELAERE (G.). Choix des échelles en cartographie des pâturages. In : Actes du colloque IICA : Inventaire et cartographie des pâturages tropicaux africains, Bamaḱ, Mali, 3-8 mars 1975, pp. 349-350, carte en coul.

17. DE WISPELAERE (G.), LAMARQUE (G.). Cartographie des pâturages naturels intertropicaux. Paris, Rev. Comité Fr. Cartographie, 1976, 67 : 12-16.

18. DE WISPELAERE (G.), TOUTAIN (B.). Estimation de l'évolution du couvert végétal en 20 ans consécutivement à la sécheresse dans le Sahel voltailque. Photo-Interprétation, 1976,15 (3), $\mathrm{n}^{\circ} 2$.

19. DE WISPELAERE (G.), TOUTAIN (B.). Un exemple de dégradation du couvert végétal dans le sahel voltaïque entre 1955 et 1975 . Photo-Interprétation, $1976,15(3), \mathrm{n}^{0} 1$.

20. GARCIA (M.). Pertes sur le bétail dues à la sécheresse en Haute-Volta. Rapport de mission. Ouagadougou, C. E. B. V., 1974.

21. GASTON (A.), DULIEU (D.) et Collab. Pâturage du Kanem, effet de la sécheresse de 1973 sur les pâturages. Comparaison avec les études de 1964 et 1966, actualisation de la carte. Maisons-Alfort I. E. M. V. T., 1976. 175 p. (Coll. Bilans régionaux).

22. GIRARD (C. M.), GIRARD (M. C.), Application de la télédétection à l'étude de la biosphère. Paris, Masson, 1975. 200 p.

23. GWYNNE (H. D.), CROZE (H.). East African habitat monitoring practic: A review of methods and application. Proc. seminar ILCA : Evaluation and mapping of tropical African rangelands, Bamako, Mali, 3-8 March 1975, pp. 95-135.

24. HEADY (H. F.). Range condition and range trend. Proc. seminar ILCA : Evaluation and mapping of tropical African rangelands, Bamako, Mali, 3-8 March 1975, pp. 177-179.

25. HODGSON (H. J.). Needs for evaluation and mapping of african rangeland. Proc. seminar ILCA : Evaluation and mapping of tropical African rangelands, Bamako, Mali, 3-8 March 1975, pp. 371-372.

26. HURAULT (J.). Surpâturage et transformation du milieu physique. Paris, I. G. N., 1975. 218 p., Photogr. (Etude de Photointerprétation $\mathrm{n}^{\circ} 7$ ).

27. HURAULT (J.). Photo-interprétation et télédétection dans le domaine du spectre visible et du proche infra-rouge. Bull. Inf. I. G. N., 1976 (32). Tribune libre: 23-37.

28. International Livestock Centre for Africa (ILCA). Colloque sur l'inventaire et la cartographie des pâturages tropicaux africains, Bamako, Mali, 3-8 mars 1975. 399 p., carte en coul.

29. LACAZE (B.), MONGET (J. M.), DULAC (J.). Correspondance analysis of multiscanner data for vegetation classification. Proc. third annual Remote Sensing of the Earth Resources Conference, Tullahoma, March 1974.

30. PEYRE DE FABREGUES (B.), ROSSETTI (C.) Evolution des pâturages naturels sahéliens du Sud Tamesna (Niger). Cartographie des potentialités fourragères par photographies aériennes. MaisonsAlfort, I. E. M. V. T., Niamey, L. N. E. R. V. ; Paris, Goetechnip, 1971, 135 p., 4 cartes en coul. (Etude agrostologique $n^{\circ} 32$ ).

31. POULTON (C. E.). A comprehensive remote sensing legend system for the ecological characterization and annotation of natural and altered landscapes. Ann Arbor, Environmental Research Inst. of Michigan. Proc., 8th. Int. Symp. on Remote Sensing of Environment, 2-6 th Oct. 1972. pp. 393-408.

32. REINING (P.). Utilization of ERTS 1 imagery in cultivation and settlement sites identification and carrying capacity estimates in Upper Volta and Niger. Final Report AID Office in accordance with the terms of AID/W contract $n^{\circ}$ AID/CM/afr. C 73.21, 1973. 155 p. 46 tabl. 32 photogr.

33. TOUTAIN (B.), DE WISPELAERE (G.). Etude et cartographie des pâturages de l'O. R. D. du Sahel et de la zone Nord de Fada N'Gourma (HauteVolta). Maisons-Alfort, I. E. M. V. T. (Etude agrostologique $n^{\circ}$ 51) (à paraître).

34. WENDEROTH (S.), YOST (E.), KALIA (R.), ANDERSON (R.). Multispectral photography for Earth resources. 2nd ed. Greenvale New York, U. S. A., Remote Sensing Information Center, 1975, 257 p., fig. 